\title{
Dyspepsia and Gut Microbiota in Female Patients with Postcholecystectomy Syndrome
}

\author{
Doina Georgescu $\mathbb{D}^{1, *}$, Alexandru Caraba $\mathbb{D}^{1, *}$, loana Ionita ${ }^{1}$, Ana Lascu $\mathbb{D}^{2}$, Emil Florin Hut ${ }^{3}$, \\ Simona Dragan $\mathbb{1}^{4}$, Oana Elena Ancusa $\mathbb{C}^{\prime}$, Ioana Suceava', Daniel Lighezan' \\ 'Department of Internal Medicine I, "Victor Babes" University of Medicine and Pharmacy, Timisoara, Romania; ${ }^{2}$ Department of Functional Sciences, \\ Discipline of Pathophysiology, "Victor Babes" University of Medicine and Pharmacy, Timisoara, Romania; ${ }^{3}$ Department IX of Surgery I/Compartment \\ of Hepato-Bilio-Pancreatic Surgery, "Victor Babes" University of Medicine and Pharmacy, Timisoara, Romania; ${ }^{4}$ Department of Cardiology, "Victor \\ Babes" University of Medicine and Pharmacy, Timisoara, Romania
}

*These authors contributed equally to this work

Correspondence: Alexandru Caraba, Department of Internal Medicine I, "Victor Babes" University of Medicine and Pharmacy, Timisoara, Piata Eftimie Murgu 2, 30004I, Romania, Tel +40 744769 850, Fax +40 256490 626, Email caraba.alexandru@umft.ro

Background: Gallstone disease (GSD) represents one of the most frequent digestive disorders, highly reported in female gender. The purpose of the study was to explore the clinical and gut microbiota particularities of female patients with postcholecystectomy syndrome (PCS) and the possible relationship between gut dysbiosis (DB) and abdominal complaints.

Patients and Methods: In total, 129 female participants: 104 outpatients divided into two equal groups, 52 PCS (+), 52 PCS (-) and 25 healthy controls were consecutively enrolled in this observational study. Patients underwent clinical examination with assessment of pain, bloating, transit disturbances, abdominal ultrasound/computer tomography/magnetic resonance imaging/endoscopic retrograde cholangiopancreatography, upper and lower digestive endoscopies. Laboratory work-ups and stool microbiology assessments were performed for all study participants (patients and controls). Stool microorganisms were identified by matrix-assisted laser desorption ionization - time-of-flight- mass spectrometry and in patients with DB also by next-generation sequencing.

Results: Older age, complicated gallstones disease, associated conditions like diabetes mellitus/impaired glucose tolerance and irritable bowel syndrome were significantly present in PCS $(+)$ group, as well as sedentary lifestyle and diets characterized by a low fiber intake $(\mathrm{p}<0.0001)$. PCS $(+)$ patients displayed significant differences related to the incidence and severity of overall gut microbiota DB, decreased H index of biodiversity and the unbalanced Firmicutes/Bacteroidetes (F/B) ratios by comparison to the PCS $(-)$ group $(\mathrm{p}<0.0001)$. Strong positive correlations of the severity of overall DB with bloating and the intestinal habit disorders, as well as of $\mathrm{F} / \mathrm{B}$ ratios to all abdominal symptoms were noted.

Conclusion: PCS in female patients was associated with older age, sedentary lifestyle, specific dietary habits, history of complicated gallstone disease, diabetes mellitus/impaired glucose tolerance and irritable bowel syndrome, as well as gut microbiota particularities. Overall DB and unbalanced F/B ratios were strongly correlated to abdominal complaints.

Keywords: postcholecystectomy syndrome, female patients, gut microbiota imbalance

\section{Introduction}

Postcholecystectomy syndrome (PCS) is a condition that represents either persistence of symptoms caused by gallbladder pathology, prior to cholecystectomy or the development of new symptoms, months to years after surgery. These symptoms encompass a large scale of abdominal complains, such as fatty food indigestion, nausea and vomiting, bloating and flatulence, diarrhea, and intermittent episodes of jaundice, or abdominal pain. Symptoms characterizing PCS may occur in 5-40\% of the cases following a cholecystectomy procedure and may manifest for a short period of time, being transitory, or last longer, sometimes the entire life span. As gallstone disease (GSD) represents one of the most prevalent digestive disorders highly reported in female gender, many cholecystectomies are performed daily all over the world in female patients and the probability of PCS development increases accordingly. ${ }^{1-3}$ It is possible for an intervention begun with the laparoscopic 


\section{Graphical Abstract}

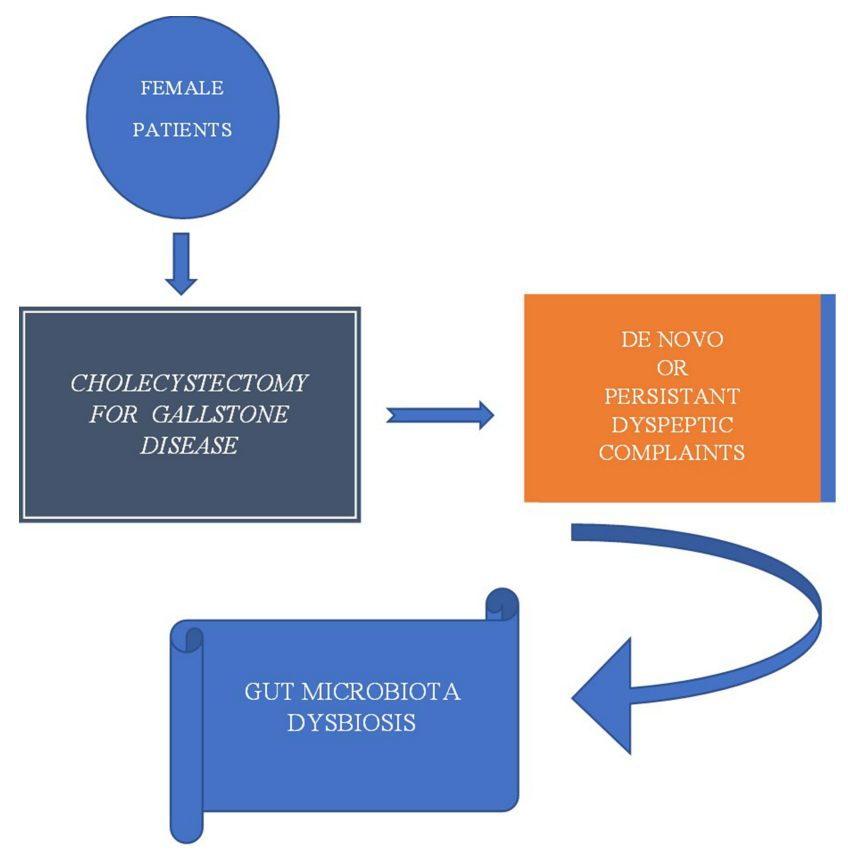

approach to be converted into the classical method and for particularities of the intervention to trigger consecutive abdominal complaints. $^{4,5}$

There are some situations that can prompt so-called prophylactic cholecystectomy. This situation is represented by adult populations with morbid obesity when performing bariatric surgery, or by patients with stones larger than $3 \mathrm{~cm}$ in diameter that could result in gallbladder (GB) carcinoma. These situations raise the question of whether prophylactic cholecystectomy could favor the development of PCS. ${ }^{6-8}$

It is difficult to preoperatively assess the risk for PCS, as no current guidelines of risk stratification are available to date. PCS can manifest in severe situations (major PCS), which often mandate reintervention or endoscopic retrograde cholangio-pancreatography (ERCP) procedures. However, other patients experience so-called minor PCS, characterized by milder abdominal complaints, such as nausea and vomiting, pain, bloating, and intestinal habit disturbances with the acceleration of intestinal transit and diarrhea. Understanding postcholecystectomy dyspepsia is not always an easy task to accomplish. Depending on the age, type, moment of intervention and major complications, patients could develop, in time, nearby issues related to duodeno-gastric and gastroesophageal reflux, dysfunction of the sphincter of Oddi, as well as surgical abdominal adhesions. ${ }^{9}$

When assessing dyspepsia, practitioners may sometimes overlook extra biliary conditions, like pancreatitis, hepatitis, or diverticulitis, that could overlap, or they may mistake several conditions for PCS. That is why, in order to correctly diagnose PCS, various conditions and diseases should first be ruled out. ${ }^{10}$ There are situations when, despite thorough explorations, one cannot identify an organic cause of these symptoms and a functional dyspepsia could come into discussion. In this case, dyspeptic complaints could develop as a consequence of local inability to adapt to GB removal, resulting in lack of bile concentration and storage, and a continuous leak of bile into the gut, without any inter-digestive pause. $^{11}$

Over the past decades, numerous researchers have contributed to understanding of the human gut microbiota and its way of influencing health or triggering pathologic processes. GSD was associated with particular gut microbiota dysbiosis not only in pigment stone pathogenesis but also in cholesterol gallstones (GS). ${ }^{12}$ There is a great probability that preexistent environmental modifications are related to gut dysbiosis, which was previously associated with GSD even after surgery and, 
therefore, present in patients with PCS. Patients with GSD, as some authors reported, displayed higher overall concentrations of fecal bile acids and decreased microbial diversity. Certain beneficial genera, such as Roseburia, appeared to be poorly represented, while others, like Oscillospira, were augmentated when compared with controls. The authors hypothesized that these genera could be used as biomarkers for symptomatic GSD. Bacteroidetes phyla were correlated to primary and secondary bile acids. ${ }^{13}$ It is, however, equally possible that new environmental conditions secondary to the GB functional absence result in reshaping the gut microbiota, as well. ${ }^{14} \mathrm{~A}$ recent study hypothesized that Proteobacteria could act as a pathogenic risk factor for dyspeptic complaints in cholecystectomized patients. ${ }^{15}$

The primary aim of this study was to explore clinical and gut microbiota particularities in female patients with PCS.

The secondary aim of this study was to assess whether there is a relation between DB and abdominal complaints in patients affected by PCS.

\section{Materials and Methods}

A cross-sectional study concerning 129 female research participants - 104 consecutive outpatients with a history of laparoscopic cholecystectomy for GSD, at least 6 months earlier and 25 healthy controls recruited from patient's siblings - was carried out. The patients were equally divided into two groups: 52 patients complaining of abdominal discomfort consistent with diagnosis of PCS and 52 patients without PCS. They joined the study after written informed consents were signed.

Between 01.05.2019 and 01.10.2020, a pool of female patients who underwent laparoscopic cholecystectomy for GSD at the General Surgery Department, University Hospital, at least 6 months prior to the commencement of this study, according to the operatory theater records, complaining or not at present of dyspeptic symptoms were checked for eligibility in the ambulatory setting. It took 484 patients to identify 52 cases with PCS that qualified for inclusion in this study. Figure 1 illustrates the inclusion flow chart of the research participants and the study group achievement. Intermediate cohort I had 164 participants, while 180 patients with H. pylori, 92 patients with gastroesophageal reflux disease (GERD), 36 with chronic viral hepatitis, 6 with chronic pancreatitis, 4 with previous gastric surgery, and 2 with inflammatory bowel disease (IBD) were not included in this study. Intermediate cohort II comprised 128 patients, in which 24 patients have been recently treated with antibiotics (ATB) and 20 patients were currently receiving probiotics (PB) to alleviate their digestive issues. The final cohort of patients that was enrolled reached 104 female patients, evenly divided as follows: 52 with PCS and 52 without PCS, due to the fact that other 24 patients did not agree to join the study.
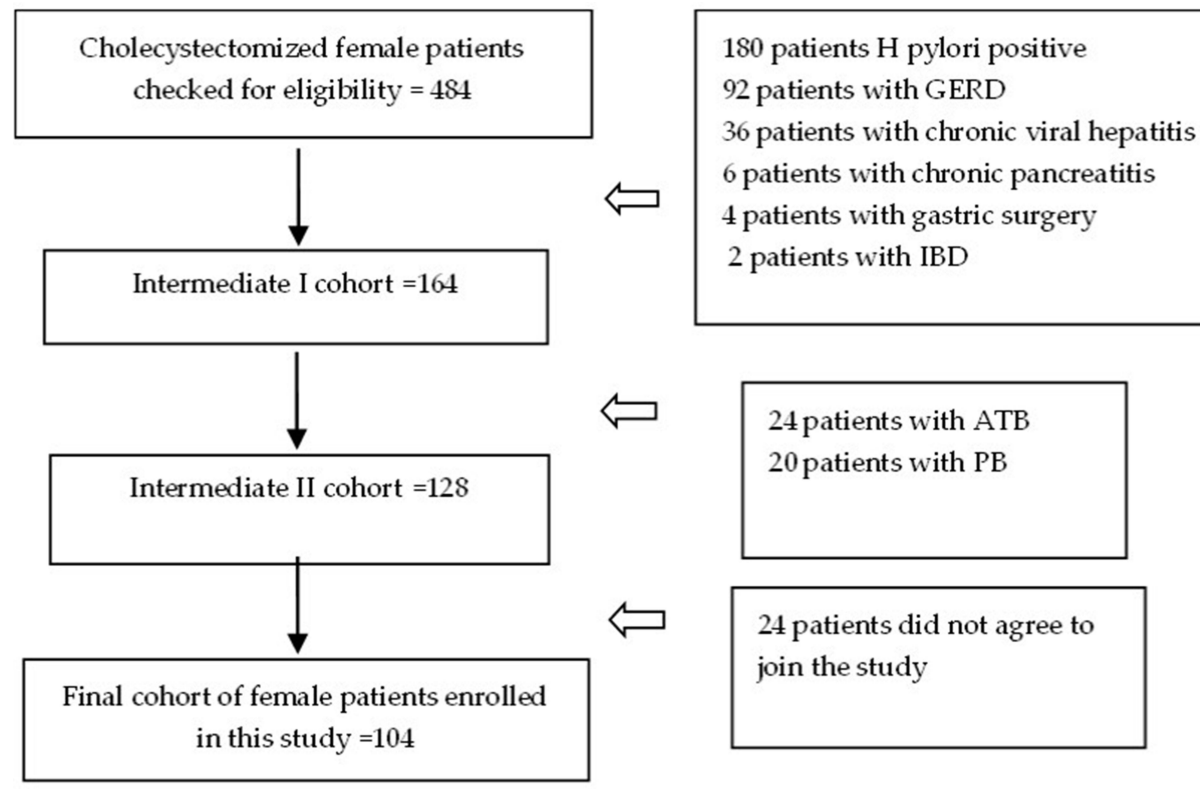

Figure I The study participants inclusion flow-chart. 


\section{Inclusion Criteria}

Female patients who underwent laparoscopic cholecystectomy for symptomatic GSD, at least 6 months earlier were consecutively included in this observational study based on the presence or absence of PCS, characterized by persistent or de novo abdominal dyspeptic complaints, such as abdominal pain, bloating, and transit disturbances. Patients were assigned to two equal groups: those complaining of PCS (study group, PCS+) and those without PCS, who did not present abdominal complaints (comparison group, PCS -).

\section{Exclusion Criteria}

Major PCS (retained or dropped calculi, biliary strictures, chronic biloma, long cystic remnant duct, and stenosis of the sphincter of Oddi); patients with organic intestinal and colonic diseases; end-stage chronic respiratory, cardiac, liver, and kidney diseases with organ failure; thyroid, oncological, and autoimmune diseases, GERD, H. pylori infection, gastroduodenitis, chronic viral or toxic hepatitis, pancreatitis, diverticulitis, jaundice; other prior abdominal surgical procedures with adhesions; patients under antibiotic or probiotic treatment at enrollment; and those who did not agree to join the study.

\section{Definitions of Various Entities}

The definition of PCS requires the recurrence of digestive symptoms similar to those experienced before cholecystectomy, principally represented by upper abdominal pain and dyspepsia with or without jaundice. ${ }^{16}$ Diabetes mellitus (DM) was diagnosed according to the American Diabetes Association (ADA) criteria. ${ }^{17}$ Dyslipidemia was confirmed according to the Panel III Guidelines, 2004. ${ }^{18}$ Arterial hypertension was diagnosed according to the National Institute for Health and Care Excellence (NICE) Guidelines 2011. ${ }^{19}$ The GS classification in cholesterol (pure/mixed) and pigment stones, used in this study was in accordance with the Japanese Society of Gastroenterology, which associates the gross morphology with the probable chemical composition of GS. ${ }^{20}$ The Rome III consensus criteria were used for the diagnosis of irritable bowel syndrome (IBS). ${ }^{21}$ Nonalcoholic fatty liver disease (NAFLD), characterized by the accumulation of fat in liver cells, in the absence of excessive alcohol consumption or any other secondary causes, resulting in steatosis or more severe liver conditions was noninvasively diagnosed using imaging examinations - ultrasound computer tomography/magnetic resonance imaging (US/CT/MRI). ${ }^{22}$ Gut microbiota DB is an entity defined by the disturbance of the normal equilibrium of the intestinal flora as a consequence of its quantitative and qualitative modifications, characterized by lack of healthy microorganisms, overgrowth of potentially dangerous microorganisms or decrease of biodiversity. ${ }^{23}$

\section{Research Participants Approach}

Patients and controls underwent a thorough clinical examination, with a history taken regarding their dietary, alcohol consumption, and cigarette smoking habits. Body mass index (BMI) assessments as well as measurements of the waist circumference and blood pressure (BP) were also performed. Gynecological consultations were also carried out in order to rule out any female pathology that could overlap the abdominal complaints. Symptoms were scored using a scale from 0 (no pain) to 6 (severe pain) for abdominal pain and from 0 (no symptoms) to 3 (severe symptoms of bloating and transit disturbances). All surgical reports of cholecystectomies were reviewed to collect data related to indications for cholecystectomy, the type of intervention (laparoscopic or conversion), number, location, and type of GS found: cholesterol or pigment stones.

Laboratory work-ups (blood, urine and stool), including a complete cell blood count, alanine-aminotransaminase, fast blood glucose, C-reactive protein, creatinine, total cholesterol triglyceride, as well as $H$. pylori fecal antigen, stool parasite examinations, and calprotectin evaluations, were performed for the patients and controls with standardized methods, accredited by Romanian Accreditation Association (RENAR) and the European Community (EC). Clinical, laboratory and imaging assessments were performed when patients have been included in this research. The study group underwent abdominal ultrasound, performed first thing in the morning, in a fasting state, using high-resolution ultrasound devices: General Electric (GE Healthcare, Chicago, IL, USA), abdominal CT/MRI (GE Healthcare), as well as gastrointestinal videoendoscopies (Olympus Europa SE\&Co.KG), electrocardiographies (ECG EDAN SE-1200 EXPRESS) with computerized 12 channels devices, thorax X-ray examinations, and, in selected cases, cholangio-CT/MRI or ERCP. 
Stool samples of all research participants (patients and controls) were collected in dedicated 30-mL sterile stool screw cap polypropylene containers for storage at $-20{ }^{\circ} \mathrm{C}$, with spoon collectors (Euro Med), then brought to the laboratory in a maximum 2-hour interval after collection. For overall gut microbiota DB assessment, progressive dilutions in saline water from $10^{-1}$ to $10^{-5}$ were obtained, and $0.1 \mathrm{~mL}$ of the stool samples was placed on the surface of plates containing different culture media for aerobe, anaerobe, and microaerophilic spp. and incubated at $37{ }^{\circ} \mathrm{C}$ for 48 hours. Matrix-assisted laser desorption ionization - time-of-flight - mass spectrometry (MALDI-TOF-MS) method was used for identifying the microorganisms. The entities found were expressed as colony formatting units (CFU)/gram stool. ${ }^{23,24}$ Dysbiosis, diagnosed as quantitative and qualitative imbalance of the intestinal flora, was assessed as $1=$ minor, $2=$ mild, $3=$ severe. ${ }^{25} 16 \mathrm{~S} \mathrm{r} \mathrm{RNA}$, nextgeneration sequencing (NGS) method was performed using frozen stools from dysbiotic patients, in order to determine the exact operational taxonomic units (OTU) of stool microbiota, based on Greengenes database. The full primer pair sequences according to standard International Union of Pure and Applied Chemistry (IUPAC) nucleotide nomenclature were 16S Amplicon PCR forward primer (50bp): 5'-TCGTCGGCAGCGTCAGATGTGTATAAGAGACAG-3' and 16S Amplicon PCR reverse primer (55 bp): 5'-GTCTCGTGGGCTCGGAGATGTGTATAAGAGACAG-3' ${ }^{26}$ The output of this entire workflow provided an elaborate presentation of different taxonomic levels from kingdom and phylum to genus and species as cluster graphs, sample tables, columns, and cluster pie charts. The final report provided information, such as the distribution of Firmicutes, Bacteroidetes, Proteobacteria, Actinobacteria, Verrucomicrobia, Fusobacteria, Euryarcheota, Tenericutes, Cyanobacteria spp. and subspp., Fungi, enterotype classifications as well as bioindicators such as the Shannon-Wiener $\mathrm{H}$ index of alpha biodiversity. ${ }^{27}$ The Firmicutes/Bacteroidetes (F/B) and Prevotella/Bacteroidetes (P/B) ratios were calculated.

This study was carried out by respecting the Helsinki declaration of human rights, and we obtained the approval of the Ethical Committee of Scientific Research with the University of Medicine and Pharmacy from Timisoara, number 21/2019.

\section{Statistical Analysis}

Statistical analysis was performed using GraphPad Prism 9.0.1 (151) version package (GraphPad Software, Inc., La Jolla, CA, USA). The continuous data panel was expressed as the mean values and standard deviations. The unpaired $t$-test was performed, and the $\mathrm{p}$ values were calculated. When $\mathrm{p}$ was $\leq 0.05$, the results were considered statistically significant, with a confidence interval $(\mathrm{CI})=95 \%$. Categorical variables were analyzed with the Chisquared test. Pearson's correlation test was performed to set the relations between variables, and graphics of the linear regression were drawn.

\section{Results}

This was an observational, cross-sectional study that included 129 female research participants: 104 cholecystectomized outpatients, divided into two equal groups, PCS (+) and PCS (-), out of 484 patients checked for eligibility and 25 healthy controls. As depicted in Table 1, we analyzed the demographic aspects and biological characteristics in the patients and controls. There were no significant differences related to the demographic variables of age and residence between the patients and controls. However, we noted significant differences regarding age between PCS (+) and PCS (-) patients, as well as between PCS $(+)$ patients and controls $(\mathrm{p}<0.0001)$. With respect to the serum biological variables, CRP from PCS $(+)$ group displayed significant differences in comparison to controls $(\mathrm{p}=0.04)$ and also fast plasma glucose was significantly increased in PCS $(+)$ group in comparison to PCS $(-)$ group $(\mathrm{p}=0.005)$ and to controls $(\mathrm{p}=0.009)$. We also noted that the PCS $(+)$ group presented a significantly higher incidence of overall gut DB $(\mathrm{p}<0.0001)$ when compared to PCS $(-)$ group and to controls.

The distribution of the GS in cholecystectomized patients, according to the composition reported at the previous surgical intervention, is illustrated in Figure 2.

As depicted in Figure 2, no significant differences were noted between the two groups. In the PCS (+) group, for the cholesterol GS, either pure or mixed stones represented the vast majority of situations and were reported in $92 \%$ of the cases, whereas pigment GS was seldom observed (8\% of cases). The PCS (-) group exhibited cholesterol GS in $78.84 \%$ of the patients $(\mathrm{p}=0.056)$ and pigment GS in $21.15 \%$ of the patients $(\mathrm{p}=0.056)$. 
Table I The Demographic and Biological Characteristics in Female Research Participants

\begin{tabular}{|c|c|c|c|c|c|c|}
\hline Variables & $\operatorname{PCS}+(n=52)$ & PCS - $(n=52)$ & Controls $(n=25)$ & pl & p2 & p3 \\
\hline Age (years) & $59.05 \pm 14.47$ & $49.1 \pm 5.21$ & $58.24 \pm 2.80$ & 0.7829 & $<0.0001 *$ & $<0.0001^{*}$ \\
\hline Location, U/R & $3|/ 2|(59.6 \mid \% / 40.39 \%)$ & $30 / 22(57.68 \% / 42.31 \%)$ & $19 / 6$ (76\%/34\%) & 0.1609 & 0.12 & 0.843 \\
\hline $\mathrm{Hb}(\mathrm{g} / \mathrm{dL})$ & $13.077 \pm 0.682$ & $13.05 \pm 0.6 \mid$ & $13.344 \pm 0.834$ & 0.1391 & 0.139 & 0.693 \\
\hline $\mathrm{L} / \mathrm{mm}^{3}$ & $(6.23 \pm 0.858) \times 10^{3}$ & $(6.5 \pm 0.79) \times 10^{3}$ & $(6.608 \pm 0.842) \times 10^{3}$ & 0.08 & 0.943 & 0.782 \\
\hline CRP (mg/dL) & $0.399 \pm 0.181$ & $0.336 \pm 0.098$ & $0.338 \pm 0.131$ & $0.04 *$ & 0.937 & 0.747 \\
\hline ALT (IU/L) & $31.89 \pm 7.01$ & $31.6 \pm 4.53$ & $30.96 \pm 5.25$ & 0.57 & 0.548 & 0.802 \\
\hline FPG (mg/dL) & $95.68 \pm 18.22$ & $87.94 \pm 6.78$ & $85.48 \pm 8.36$ & $0.009 *$ & 0.163 & $0.005^{*}$ \\
\hline T Chol (mg/dl) & $204.21 \pm 30.92$ & $201.48 \pm 18.50$ & $192.88 \pm 7.7$ & 0.0759 & 0.143 & 0.632 \\
\hline Trig (mg/dL) & $|43.9| \pm 26.98$ & $137.88 \pm 30.64$ & $132.56 \pm 10.07$ & 0.1220 & 0.402 & 0.282 \\
\hline $\mathrm{Cr}(\mathrm{mg} / \mathrm{dL})$ & $0.68 \pm 0.09$ & $0.736 \pm 0.121$ & $0.69 \pm 0.11$ & 0.5929 & 0.682 & 0.286 \\
\hline Overall gut DB & $43 / 52$ (82.68\%) & $7 / 52$ (13.4\%) & $2 / 25(8 \%)$ & $<0.0001 *$ & 0.4920 & $<0.000 I^{*}$ \\
\hline
\end{tabular}

Note: *Statistically significant.

Abbreviations: PCS, postcholecystectomy syndrome; n, number; pI, PCS (+) vs controls; P2, PCS (-) vs controls; p3, PCS (+) vs PCS (-); M/F, males/females; U/R, urban/ rural; Hb, hemoglobin; L, leukocytes; CRP, C-reactive protein; ALT, alanine-aminotranspherase; FPG, fast plasma glucose; T Chol, total cholesterol; trig, triglycerides; cr, creatinine; DB, dysbiosis; g/dL, grams/deciliter; mm, millimeter; $\mathrm{mg} / \mathrm{dL}$, milligrams/deciliter; IU/L, international units/liter.

The gut microbiota DB aspects in the cholecystectomized female patients (PCS + and PCS - ) are depicted in Table 2, where the PCS (+) group exhibited significantly higher incidence and severity of the overall DB of the gut microbiota, as well as unbalanced F/B ratios and decreased $\mathrm{H}$ index of biodiversity, by comparison to the PCS (-) group $(\mathrm{p}<0.0001)$.

Table 3 illustrates various aspects related to the clinical particularities in patients: PCS (+) vs PCS (-).

As seen in Table 3, by comparing the PCS $(+)$ patients to the PCS (-) patients, the study group displayed multiple particularities. Interventions performed for complicated GSD were reported in significantly larger proportion of PCS $(+)$ patients (more than $75 \%$ ), $\mathrm{p}<0.0001$. A total of $59.61 \%$ of the PCS $(+)$ patients presented a sedentary lifestyle, by comparing to the PCS (-) patients who exhibited the same lifestyle only in $38.46 \%(\mathrm{p}=0.03)$. The diet characterized by a low fiber intake was noted in significantly larger proportion $(52.83 \%)$ of PCS $(+)$ patients $(\mathrm{p}=0.003)$. Statistically significant differences were observed in favor of the PCS $(+)$ patients who exhibited DM/IGT and IBS in higher proportion $(\mathrm{p}<0.0001)$. No significant differences were noted related to the average time since cholecystectomy, alcohol consumption, cigarettes smoking, history of oral contraception, multiparity, postmenopausal hormone replacement therapy, intake of saturated fat and carbohydrates. or various associated conditions such as metabolic conditions (obesity,

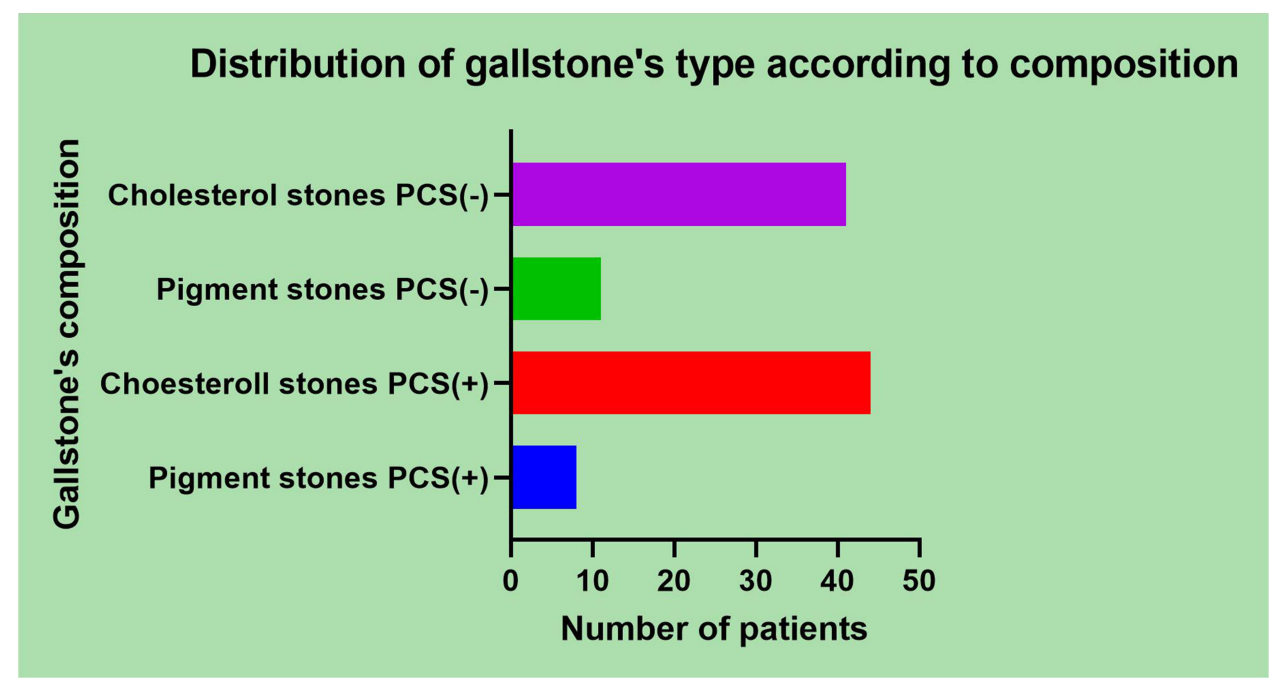

Figure 2 Distribution of the previous gallstone's type in cholecystectomized female patients. 
Table 2 Dysbiosis Characteristics in Female Cholecystectomized Patients

\begin{tabular}{|l|l|l|l|}
\hline Variables & \multicolumn{1}{|c|}{ PCS + (n=52) } & \multicolumn{1}{|c|}{ PCS - (n=52) } & \multicolumn{1}{|c|}{ P } \\
\hline Incidence of overall DB & $82.69 \%$ & $13.4 \%$ & $<0.000 I^{*}$ \\
Overall DB score & $1.46 \pm 0.89$ & $0.17 \pm 0.47$ & $<0.000 I^{*}$ \\
Imbalance of F/B & $69.23 \%$ & $9.61 \%$ & $<0.000 I^{*}$ \\
Increased F/B & $9.61 \%$ & $0 \%$ & $0.0226 *$ \\
Decreased F/B & $59.62 \%$ & $9.61 \%$ & $<0.000 I^{*}$ \\
F/B & $23.54 \pm 94.7$ & $1.18 \pm 0.14$ & 0.5386 \\
P/B & $0.67 \pm 1.03$ & $0.57 \pm 1.17$ & 0.816 \\
Shannon-Wiener H index & $2.87 \pm 0.34$ & $2.79 \pm 0.28$ & 0.584 \\
Decreased Shannon- Wiener H index & $36.53 \%$ & $5.76 \%$ & $<0.0001 *$ \\
\hline
\end{tabular}

Note: *Statistically significant.

Abbreviations: PCS, postcholecystectomy syndrome; n, number; DB, dysbiosis; I, imbalance; F/B, Firmicutes/Bacteroidetes; P/B, Prevotella/ Bacteroidetes; DB, dysbiosis.

Table 3 Clinical Characteristics in Cholecystectomized Female Patients: PCS (+) and PCS (-)

\begin{tabular}{|c|c|c|c|c|}
\hline Variables & \multicolumn{2}{|c|}{$\operatorname{PCS}(+)(n=52)$} & $\operatorname{PCS}(-)(n=52)$ & $\mathbf{p}$ \\
\hline Time since cholecystectomy (months) & \multicolumn{2}{|l|}{$12.15 \pm 3.96$} & $11.71 \pm 3.16$ & 0.5325 \\
\hline Conversion & \multicolumn{2}{|l|}{$11.53 \%$} & $3.34 \%$ & 0.1131 \\
\hline Complicated GSD at intervention & \multicolumn{2}{|l|}{$76.92 \%$} & $40.38 \%$ & $0.0002^{*}$ \\
\hline \multirow[t]{2}{*}{ Dyspepsia } & Persistent & $79.24 \%$ & - & - \\
\hline & De novo & 20.76 & - & - \\
\hline Smoking history & \multicolumn{2}{|l|}{$48.07 \%$} & $34.61 \%$ & 0.1654 \\
\hline Alcohol consumption & \multicolumn{2}{|l|}{$13.46 \%$} & $9.61 \%$ & 0.5408 \\
\hline History of oral contraception & \multicolumn{2}{|l|}{$40.38 \%$} & $34.61 \%$ & 0.6180 \\
\hline Multiparity & \multicolumn{2}{|l|}{$32.7 \%$} & $26.93 \%$ & 0.2642 \\
\hline Postmenopausal hormone therapy & \multicolumn{2}{|l|}{$23.08 \%$} & $13.46 \%$ & 0.2065 \\
\hline Sedentary lifestyle & \multicolumn{2}{|l|}{$59.61 \%$} & $38.46 \%$ & $0.0318^{*}$ \\
\hline \multirow[t]{2}{*}{ Fiber's intake } & Normal & $48.07 \%$ & $73.07 \%$ & $0.0094 *$ \\
\hline & Reduced & $53.84 \%$ & $26.93 \%$ & $0.0054^{*}$ \\
\hline \multirow[t]{2}{*}{ Saturated fat intake } & Normal & $67.3 \%$ & $76.92 \%$ & 0.2764 \\
\hline & Increased & $32.7 \%$ & $23.08 \%$ & 0.6063 \\
\hline \multirow[t]{2}{*}{ Carbohydrate's intake } & Normal & $84.61 \%$ & $90.38 \%$ & $0.376 I$ \\
\hline & Increased & $15.39 \%$ & $9.62 \%$ & $0.376 I$ \\
\hline $\mathrm{BMI}>30 \mathrm{~kg} / \mathrm{m}^{2}$ & \multicolumn{2}{|l|}{$51.92 \%$} & $40.38 \%$ & 0.2401 \\
\hline T2DM/IGT & \multicolumn{2}{|l|}{$65.38 \%$} & $15.38 \%$ & $<0.000 I^{*}$ \\
\hline Dyslipidemia & \multicolumn{2}{|l|}{$40.38 \%$} & $28.84 \%$ & 0.2183 \\
\hline $\mathrm{AH}$ & \multicolumn{2}{|l|}{$38.46 \%$} & $28.84 \%$ & 0.3015 \\
\hline Abdominal pain score & \multicolumn{2}{|l|}{$2.17 \pm 2.39$} & - & - \\
\hline Bloating score & \multicolumn{2}{|l|}{$1.32 \pm 0.73$} & - & - \\
\hline Transit disorder score & \multicolumn{2}{|l|}{$1.44 \pm 0.69$} & - & - \\
\hline IBS & \multicolumn{2}{|l|}{$67.3 \%$} & $23.07 \%$ & $<0.000 I^{*}$ \\
\hline NAFLD & \multicolumn{2}{|l|}{$46.15 \%$} & $38.46 \%$ & 0.4296 \\
\hline
\end{tabular}

Note: *Statistically significant.

Abbreviations: PCS, postcholecystectomy syndrome; n, number; GSD, gallstone disease; BMI, body mass index; kg/m², kilogram/square meter; T2DM/IGT, type 2 diabetes mellitus/impaired glucose tolerance; AH, arterial hypertension; IBS, Irritable bowel syndrome; NAFLD, nonalcoholic fatty liver disease.

and dyslipidemia), cardiovascular conditions (arterial hypertension), and liver conditions, like NAFLD. Related to the dyspeptic complaints of the PCS (+) group of patients, the vast majority (almost $80 \%$ ) presented a persistent dyspepsia, while only $20.76 \%$ reported de novo symptoms. 
The correlation study revealed that the overall DB score in PCS (+) group was positively strongly correlated to bloating $\left(r^{2}=9.47\right)$ and transit disturbances $\left(r^{2}=0.43\right), \mathrm{p}<0.0001$, while the correlation to abdominal pain was relatively weak: $r^{2}=0.06, \mathrm{p}=0.07$, and no correlations were set to the average time (months) since cholecystectomy $\left(r^{2}=0.004\right.$, $\mathrm{p}=0.64$ ), as depicted in Figure 3.

The enterotypes in PCS (+) were determined based on the proportional relationship between Bacteroides spp., Prevotella spp., and Ruminococcus spp. as depicted in Figure 4, where one can observe that 17 cases were classified as type $1(38.53 \%), 6$ cases were classified as type $2(13.95 \%), 1$ case as type $3(2.32 \%)$, and 19 cases as undetermined (44.18\%).

The distribution of bioindicators, such as the Shannon-Wiener $\mathrm{H}$ index of alpha biodiversity, F/B, and P/B in dysbiotic patients from PCS (+) group is illustrated in Figure 5. We found that $67.3 \%$ of dysbiotic, PCS $(+)$ patients displayed an $\mathrm{H}$ index above 2.8 , and about $32.7 \%$ displayed a decreased index. The F/B ratio ranged between 0.5 and 569 , with increased values over 2.1 in $7.69 \%$ of the patients and decreased values under 1.4 in $65.38 \%$ of the patients. The $\mathrm{P} / \mathrm{B}$ ratio ranged between 0.1 and 3.5 ; a ratio over 1.8 was present in $13.95 \%$ of the patients.

The distribution of bacterial strains in dysbiotic, PCS $(+)$ patients is illustrated in Figure 6. As seen in this figure, Firmicutes ranged between 31.03 and 94.58, Bacteroidetes from 0.14 to 66.06, Proteobacteria from 0.57 to 32.35 , Actinobacteria from 0.000 to 20.48 , Verrucomicrobia from 0.001 to 9.59 , Fusobacteria from 0.003 to 1.27 , Euryarcheota from 0.000 to 0.04 , and Tenericutes from 0.001 to 17.02 .

As illustrated in Figure 7, in the PCS $(+)$ group, Firmicutes was strongly correlated to transit disturbances $(\mathrm{p}=0.0002$, $\left.r^{2}=0.28\right)$, had a good correlation to bloating $\left(\mathrm{p}=0.03, r^{2}=0.1\right)$ and a low correlation to abdominal pain $\left(\mathrm{p}=0.05, r^{2}=0.08\right)$.

As depicted in Figure 8, Bacteroidetes displayed strong correlations to transit disturbances $\left(r^{2}=0.38, \mathrm{p}<0.0001\right)$, good correlations to bloating $\left(\mathrm{p}=0.01, r^{2}=0.12\right)$, and low correlations to abdominal pain $\left(r^{2}=0.08, \mathrm{p}=0.05\right)$, in the PCS $(+)$ dysbiotic patients.

As seen in Figure 9, we illustrated the correlations between the F/B ratio and abdominal complaints in PCS (+) dysbiotic patients. Strong correlations were observed between the F/B ratio and abdominal pain $\left(\mathrm{p}<0.0001, r^{2}=0.34\right)$, as well as transit disturbances $\left(\mathrm{p}=0.0025, r^{2}=0.20\right)$, and bloating $\left(\mathrm{p}=0.0078, r^{2}=0.16\right)$.

Proteobacteria, Verrucomicrobia, Fusobacteria, Cyanobacteria, Euryarcheota, and Tenericutes, as well as the P/B ratios did not show any correlations to dyspeptic symptoms, while Actinobacteria displayed good positive correlations only to abdominal pain $\left(r^{2}=0.11, \mathrm{p}=0.024\right)$, in PCS $(+)$ dysbiotic patients, as illustrated in Figure 10.

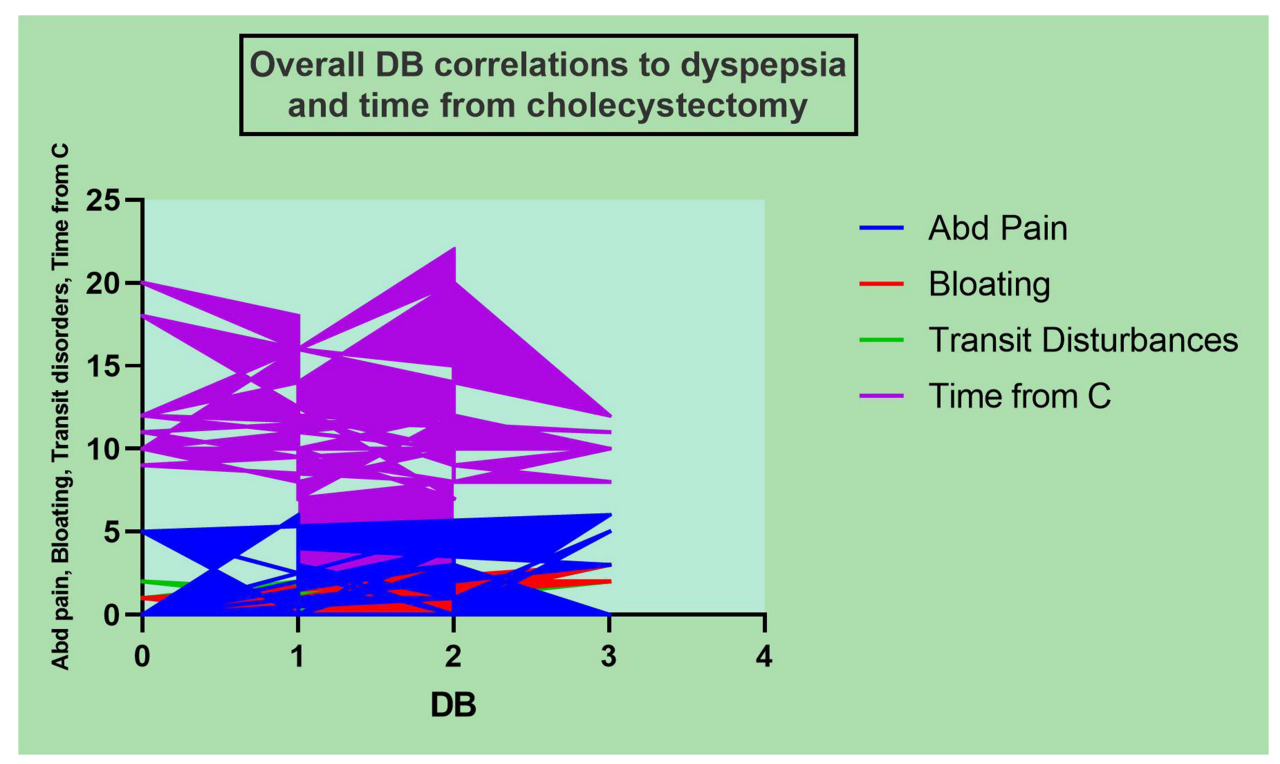

Figure 3 Correlations of the overall DB severity to the intensity of abdominal complaints and average time from cholecystectomy, in PCS (+) group. 


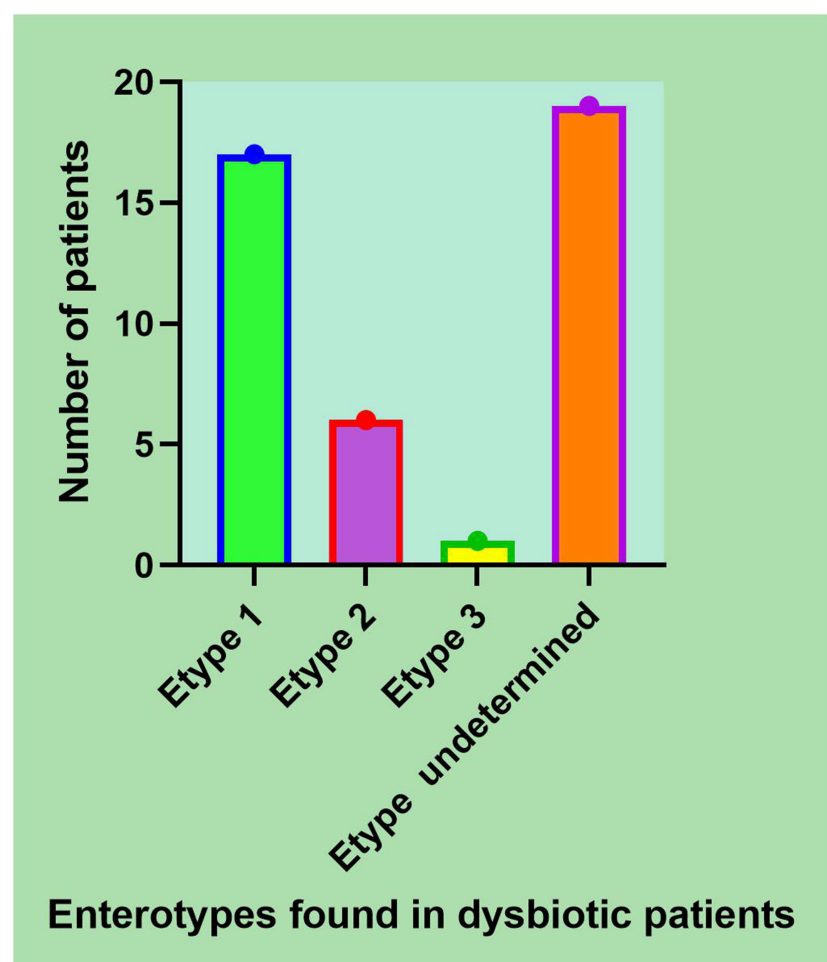

Figure 4 Distribution diagram of the enterotypes in dysbiotic, PCS (+) patients.

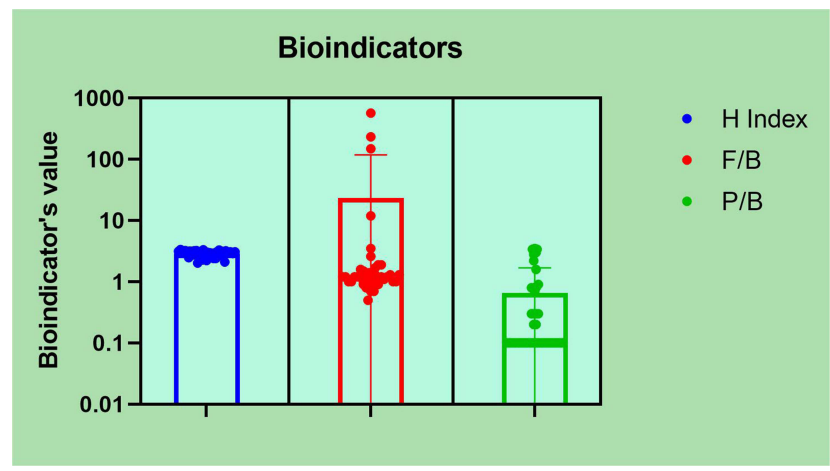

Figure 5 Distribution diagram of the bioindicators: $\mathrm{H}$ index, F/B, and P/B.

As seen in Figure 11, in the PCS $(+)$ group, the H index of biodiversity had a low correlation to abdominal pain $\left(\mathrm{p}=0.05, r^{2}=0.08\right)$ and did not correlate to the rest of the dyspeptic complaints.

\section{Discussion}

Minimizing the PCS is an important task for surgeons when it comes to performing a cholecystectomy for GSD. A complete preoperative evaluation as well as choosing the perfect moment of intervention in the course of GSD, "not too soon and not too late", could influence the postoperative outcome of patients. Urgent surgery and its associate complications will increase the risk of PCS development, as well as the increase of the duration of preoperative symptoms will also result in augmented risk of PCS. ${ }^{28}$ In accordance with this observation, we also found that more than $75 \%$ of the cases, from PCS (+) group were operated on for various complications of GSD, which also increased the rate of conversion to open surgery. 


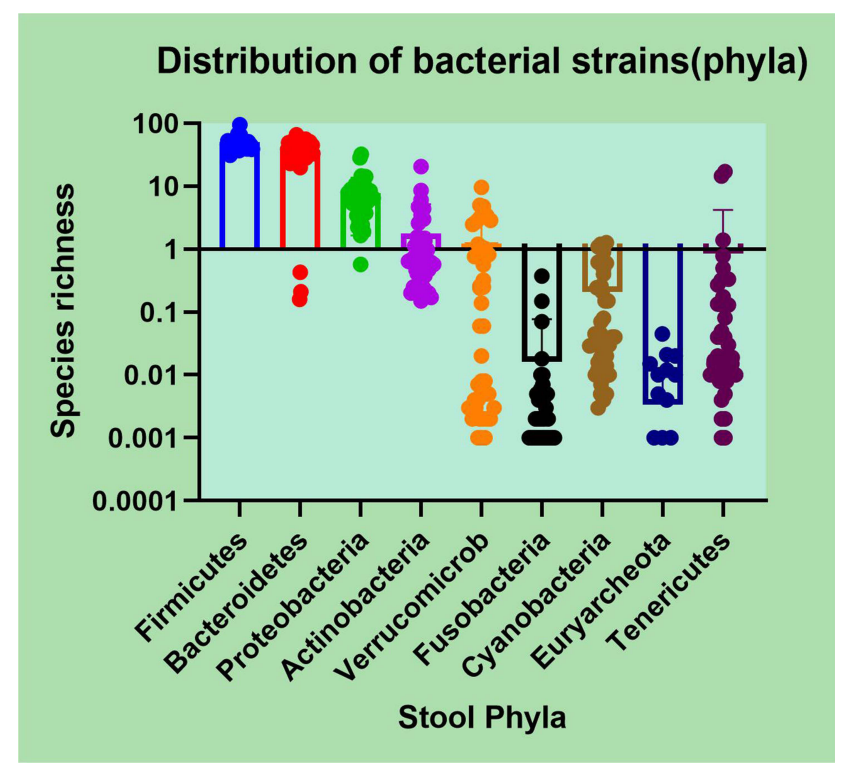

Figure 6 The distribution of bacterial strains in the dysbiotic PCS (+) population.

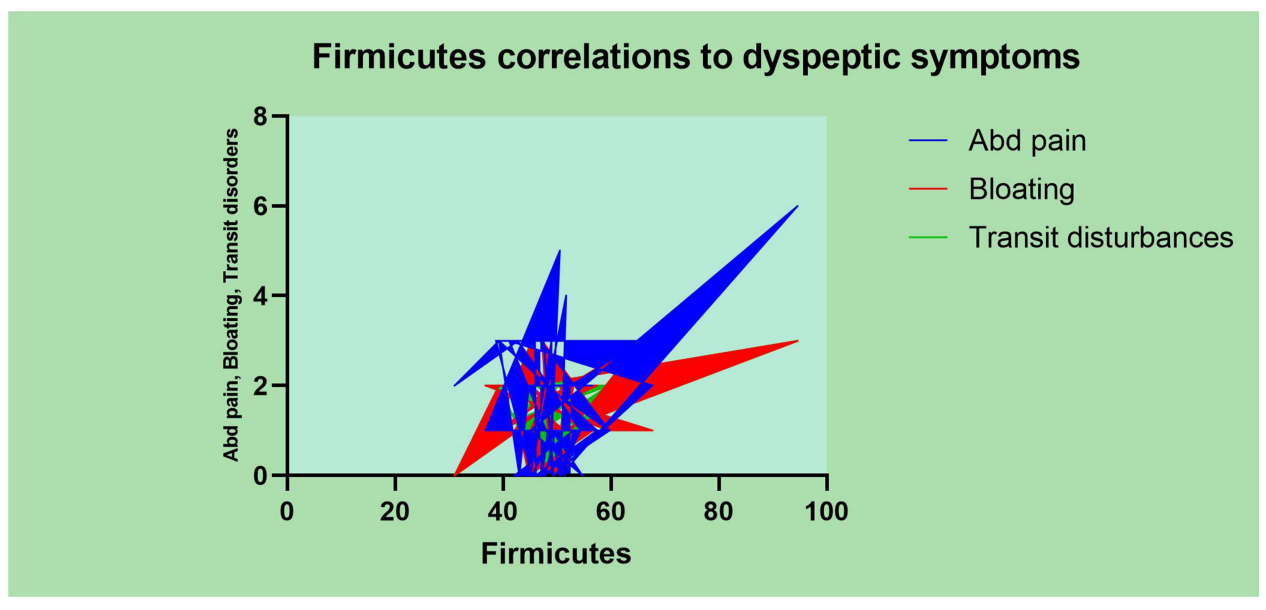

Figure 7 The Firmicutes correlations to dyspeptic symptoms.

Could the type of surgical intervention influence the PCS development? In this series, all patients experienced laparoscopic cholecystectomies. It is well known that the laparoscopic cholecystectomies have a lot of advantages over the classical open surgery, at least related to the management of inflammation and sepsis and subsequently to the hospitalization duration and to the recovery time. However, it is difficult to assess whether during dissection of the cholecystic pedicle all surgeons have really obtained the "so-called" critical view of safety, when the cholecystectomy was performed by laparoscopic approach. ${ }^{29,30}$

This research revealed that many of the patients from the study group, PCS (+), suffered at present from persistent dyspeptic complaints and functional digestive conditions. It is difficult to clearly state whether these conditions preceded surgery or developed after. Overlapping symptoms, such as discomfort, bloating, and transit disturbances prompt the question: what if IBS associated with GSD was previously overlooked, and dyspeptic symptoms were misattributed to GSD? We noted that IBS was present in an important, statistically significant proportion of the study group. The presence 


\section{Bacteroidetes correlations to dyspeptic symptoms}

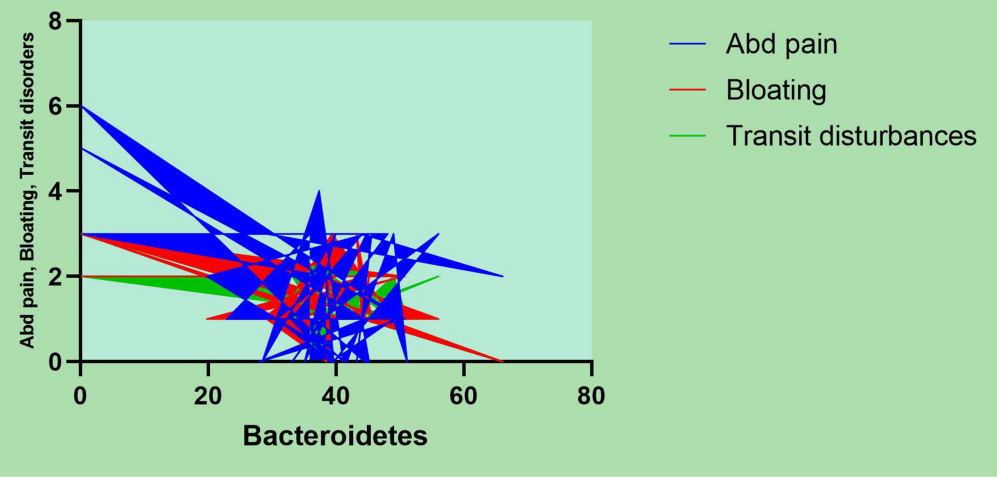

Figure 8 The Bacteroidetes correlations to dyspeptic symptoms.

of associated IBS in such a large proportion could further explain why we detected such an important range of DB as others also reported. ${ }^{31-33}$

The present study also recorded that metabolic issues associated to PCS were rather important. Many patients presented lifestyle particularities and were affected by obesity, diabetes, dyslipidemia, and hypertension, conditions

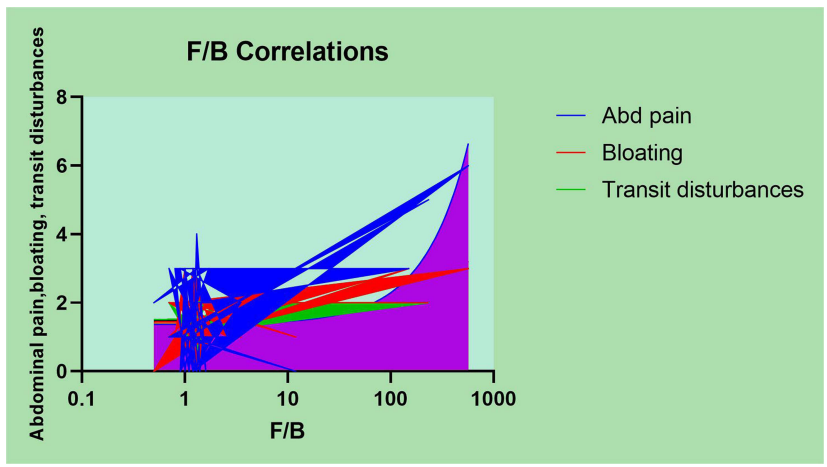

Figure 9 Correlations of the F/B ratio to dyspeptic symptoms.

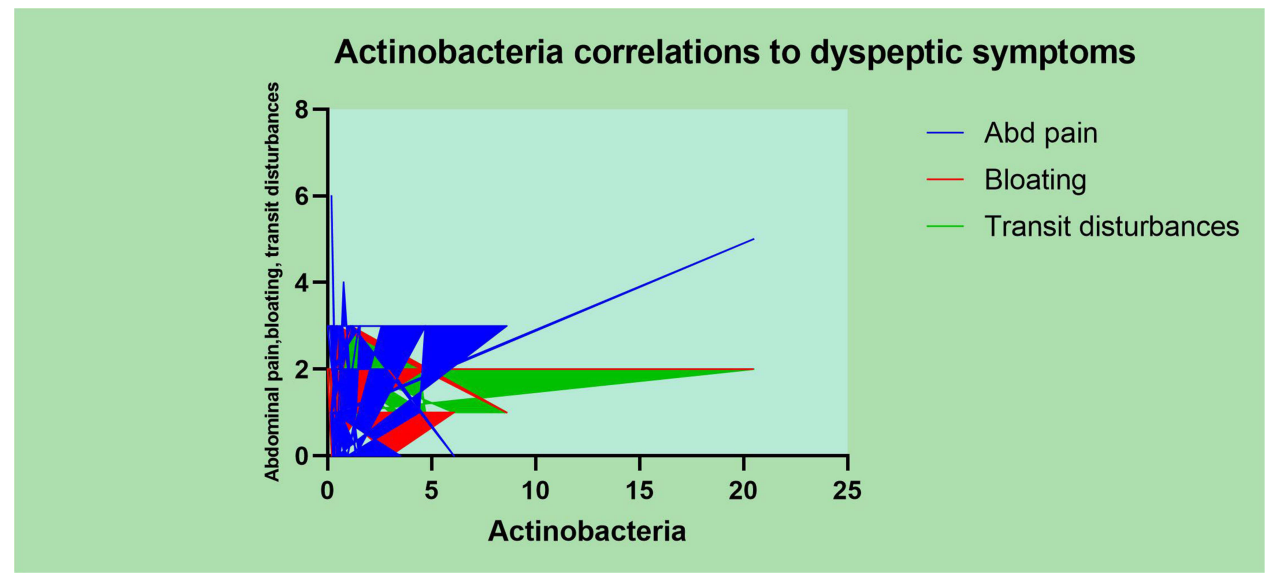

Figure 10 Actinobacteria correlations to dyspeptic symptoms. 


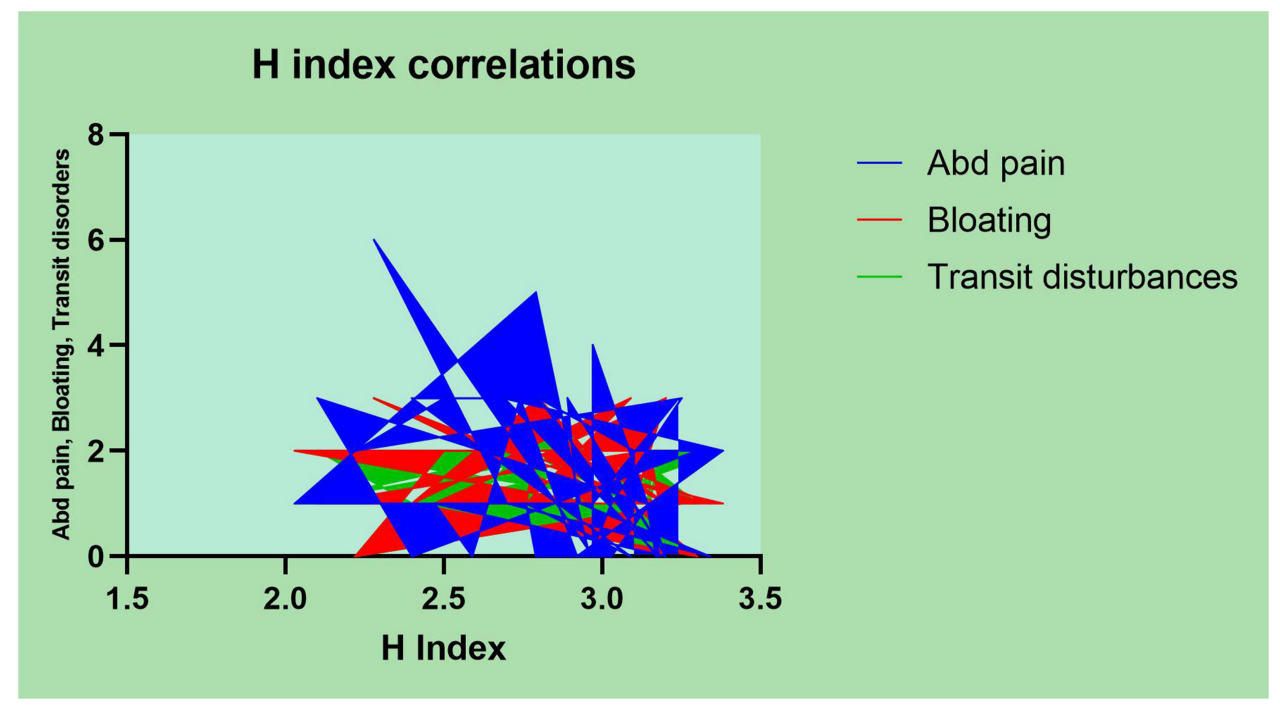

Figure II The Shannon-Wiener H index of biodiversity correlations to dyspeptic symptoms.

known for their link to gut DB. ${ }^{34-37}$ In patients with previous cholesterol GSD, it is possible that persisting underlying metabolic issues fuel the gut DB, causing the gut microbiota DB to continue or even to aggravate after the surgery. Even it is difficult to assess the exact causality of gut DB; however, the fact that the percentage of dysbiotic patients exceeded the percentage of diabetics by far provides a clue regarding postcholecystectomy gut microbiota development.

DB of the gut microbiota could intervene in many processes with the development of functional distress, or by triggering or aggravating organic, degenerative disorders at different levels. ${ }^{38-45}$ The GS composition was also associated with the particularities of the gut microbiota characterized by decreases in the biodiversity and Firmicutes. ${ }^{46-48}$ Our investigation concerning the overall DB of the gut microbiota revealed that patients with PCS displayed a significantly higher incidence and severity of DB compared to the PCS (-) group and the controls. We observed that the overall DB had a strong correlation to bloating and transit disturbances, had a low correlation to abdominal pain, and did not correlate to the time since the cholecystectomy. The vast majority of patients had previous cholesterol GS, and this study reported a high rate of dysbiosis and a microbiota footprint characterized by a decrease in the F/B ratio and diversity after cholecystectomy.

The bioindicators analyzed in this study were the F/B and $\mathrm{P} / \mathrm{B}$ ratios, as well as the Shannon-Wiener $\mathrm{H}$ index of alpha biodiversity. Only the F/B ratio correlated to all dyspeptic symptoms, while the $\mathrm{P} / \mathrm{B}$ ratio did not show any correlations, and the $\mathrm{H}$ index had only a low correlation to abdominal pain. Firmicutes and Bacteroidetes, separately analyzed, demonstrated a strong or good correlation to dyspeptic complaints, while Actinobacteria presented a good correlation only to abdominal pain, and the other phyla (Proteobacteria, Verrucomicrobia, Fusobacteria, Cyanobacteria, Euryarcheota, and Tenericutes) did not correlate to any of the dyspeptic complaints. Assessment of the proportional relationship between Bacteroides, Prevotella, and Ruminococcus spp. permitted to classify the study group in three known enterotypes as follows: more than $50 \%$ of the patients displayed type $1(38.53 \%)$ and type $2(13.95 \%)$. The rest were type $3(2.32 \%)$, and $44.18 \%$ presented undetermined enterotypes. The Bacteroides and Prevotella enterotypes, mainly observed in this study, were associated with a diet rich in saturated fat and animal protein and reduced of intake of dietary fibers as others previously reported. ${ }^{49-51}$ This microbiota footprint could explain the association to the cholesterol GSD as well as to various underlying metabolic conditions that were observed in the patients enrolled in this study. We noted that NAFLD, associated to other metabolic issues, such as obesity DM/ITG and dyslipidemia, was frequently diagnosed in patients suffering from PCS (46.15\%). As many studies hypothesized, NAFLD, inflammation, and the gut DB appear to be related. ${ }^{52,53}$ 
It is well known that GSD, cholecystectomies and by consequence the risk for PCS are by far highly reported in female gender, but is there any relation between age at intervention and PCS development? While some authors have reported age-related pre-, intra- and postcholecystectomy differences, highlighting the fact that the elderly people were predisposed to develop various complications, including PCS, other researchers did not find any clear evidence supporting the idea of high risk for PCS in older age. ${ }^{54,55}$ The results of the present study have shown that PCS (+) group displayed significantly older age by comparison to those without PCS.

Among the stimuli that can drive bioenergetic pathways and the activity of mitochondria as well as the host immunometabolism with consecutive reactions of the effector cells, the gut microbiota DB has a pivotal place. It is possible that DB to shift the capacity of the adaptative host response results in a proinflammatory state as a consequence of releasing various interleukins and cytokines. ${ }^{35,56,57}$ This study also reports a significant increase of acute phase reactant proteins, such as CRP in patients with PCS, when compared to the control group.

There are many studies highlighting the risk for colorectal cancer, postcholecystectomy, that hypothesized the possible implications of certain particularities of the gut microbiota. Some authors either reported absent intestinal bacteria, such as Bacteroides, while others described imbalances of microbiota with increased Bacteroides ovatus, Prevotella copri, and Fusobacterium varium and decreased of Faecalibacterium prausnitzii, Roseburia faecis, and Bifidobacterium adolescentis. ${ }^{58,59}$ In this series of patients, we did not find any cases of colorectal cancer, possibly related to the fact that the average time since cholecystectomy was a maximal 22 months in the PCS (+) group. We observed, however, that patients from the study group, where DB was significantly higher, displayed inflammatory markers with higher levels of CRP, which could be associated with risk for further developments as others reported. ${ }^{60,61}$

Many researches highlighted that increase in using of antibiotics over the past decades could result in important issues related to gut microbiota dysbiosis. These changes in gut ecology consist of several modifications of quantity and quality of various species, decreasing of biodiversity, alteration of metabolic functions and selection of several antibioticresistant organisms. ${ }^{62,63}$ Given the fact that a significant higher percentage of patients with PCS had a history of inflammation or infection of the gallbladder, it is also possible that antibiotics previously given for complicated GSD could have intervened in the setting of severe dysbiosis in these groups of patients.

PCS therapy remains somewhat disappointing. Except the cases that could be managed by minimally invasive procedures, or reintervention in severe situations, for the rest-representing the vast majority-there are no current guidelines of conservative treatment in addition to recommendations, such as dietary and lifestyle changes, antispasmodics, and sedatives or cholestyramine for those with accelerated transit alone. ${ }^{64-66}$

In the past, the impact of cholecystectomy on health was insufficiently understood. Over the past years, several studies highlighting the role of gut microbiota gave the possibility to associate postcholecystectomy dysbiosis to gastrointestinal symptoms such as dyspepsia and diarrhea.

Since the study of the gut microbiota DB in patients with PCS could result in new promising management of abdominal complaints and customized therapies, it is important that these preliminary observations are further validated in larger prospective studies. In this view, the hypothesis of DB involvement in symptoms related to PCS with better dyspepsia understanding could produce interesting alternative treatments as a result of translational medicine achievements. When it comes to clinical application of knowledge about the role of gut dysbiosis in the pathogenesis of postcholecystectomy syndrome, the treatment with intestinal pre/probiotics and microecological agents could result in restoring the balance of local microbiota and eventually alleviation of abdominal symptoms. ${ }^{15,67}$

This observation could considerably improve the management of dyspeptic complaints in patients with PCS, depending on the stool microbiology charts, to recommend customized probiotics or synbiotics.

\section{Limitations}

The limitations of this work include the relatively small sized sample of the study population and possible confounders, given the difficulty to discriminate the causality of gut DB, as many patients already had type 2 DM and IBS. Another confounder of dysbiosis etiopathogenesis could be associated to previously course of antibiotics, related to hospitalization in surgery department, aiming at managing infectious complication of GSD. Other statistical bias and potential drawbacks could also have intervened, given the observational study design. That is why these observations should be 
further validated by larger prospective studies. To fully realize the potential of cholecystectomy, we need to make further careful studies on the alterations of gut microbiota in patients with PCS, which may provide new ideas for the study of related-diseases after cholecystectomy and new treatment strategies for PCS.

\section{Conclusions}

Female patients with PCS displayed clinical particularities such as older age, interventions performed for complicated gallstone disease, associated conditions like diabetes mellitus/impaired glucose tolerance and irritable bowel syndrome, as well as sedentary lifestyle and specific dietary habits. Gut microbiota overall DB and unbalanced F/B ratios were often observed in patients with PCS and were strongly correlated to abdominal complaints. Understanding the relation between dyspepsia and gut DB could provide customized therapies, according to the microbiological charts.

\section{Acknowledgments}

The authors would like to express their gratitude to "Bioclinica" Laboratories from Timisoara, for technical support.

\section{Disclosure}

The authors declare no conflicts of interest in this work.

\section{References}

1. Filip M, Saftoiu A, Popescu C, et al. Postcholecystectomy syndrome: an algoritmic approach. J Gastrointestin Liver Dis. 2009;18(1):67-71.

2. Stinton ML, Shaffer EA. Epidemiology of gallbladder disease: cholelithiasis and cancer. Gut Liver. 2012;6(2):172-187. doi:10.5009/ gnl.2012.6.2.172

3. Schofer JM, Schofer MJ. Biliary causes of postcholecystectomy syndrome. J Emerg Med. 2010;39(4):406-410. doi:10.1016/j. jemermed.2007.11.090

4. Portincasa P, Moschetta A, Petruzzelli M, Palasciano G, Di Ciaula A, Pezzolla A. Gallstone disease: symptoms and diagnosis of gallbladder stones. Best Pract Res Clin Gastroenterol. 2006;20:1017-1029. doi:10.1016/j.bpg.2006.05.005

5. Radu D, Olariu S, Marinescu A, Georgescu D, Teodorescu M. Laparoscopic cholecystectomy, rate and predictors for conversion. Surg Endosc. 2011;25:54-148.

6. Cabarrou P, Portier G, Chalret Du Rieu M. Prophylactic cholecystectomy during abdominal surgery. J Visceral Surg. 2013;350(4):229-235. doi:10.1016/j.jviscsurg.2013.06.003

7. Gupta SK, Shukla VK. Silent gallstones: a therapeutic dilemma. Trop Gastroenterol. 2004;25(2):65-68.

8. Venneman NG, Buskens E, Besselink MG, et al. Small gallstones are associated with increased risk of acute pancreatitis: potential benefits of prophylactic cholecystectomy? Am J Gastroenterol. 2005;100(11):2540-2550. doi:10.1111/j.1572-0241.2005.00317.x

9. Danley T, St Anna L. Postcholecystectomy diarrhea: what relieves it? J Fam Pract. 2011;60(10):632.

10. Lamberts MP, Lugtenberg M, Rovers MM, et al. Persistent and de novo symptoms after cholecystectomy: a systematic review of cholecystectomy effectiveness. Surg Endosc.2013; 27(3):709-718.

11. Schmidt M, Sondenaa K, Dumot JA, et al. Post-cholecystectomy symptoms are caused by persistence of a functional gastro-intestinal disorder. WJGl. 2012;18(12):365-1372.

12. Wu T, Zhang Z, Liu B, et al. Gut microbiota dysbiosis and bacterial community assembly associated with cholesterol gallstones in large scale study. BMC Genom. 2013;14:669. doi:10.1186/1471-2164-14-669

13. Keren N, Kornicoff FM, Paitan Y, et al. Interactions between intestinal microbiota and bile acids in gallstones patients. Environ Microb Rep. 2015;7 (6):874-880. doi:10.1111/1758-2229.12319

14. Yoon WJ, Kim H-N, Park E, et al. The impact of cholecystectomy on the gut microbiota: a case-control study. J Clin Med. $2019 ; 8(1): 79$. doi: $10.3390 / \mathrm{jcm} 8010079$

15. Kang Z, Lu M, Jiang M, Zhou D, Huang H. Proteobacteria acts as a pathogenic risk-factor for chronic abdominal pain and diarrhea in post-cholecystectomy syndrome patients: a gut microbiome metabolomics study. Med Sci Monit. 2019;25(25):7312-7320. doi:10.12659/ MSM.915984

16. Jaunoo SS, Mohandas S, Almond LM. Postcholecystectomy syndrome (PCS). Int J Surg. 2011;8(1):15-17. doi:10.1016/j.ijsu.2009.10.008

17. American Diabetes Association. Diagnostic and classification of diabetes mellitus. Diabetes Care. 2014;37(supp11):S81-S90. doi:10.2337/dc14S081

18. Grundy SM, Cleeman JI, Merz CNB, et al. Implications of recent clinical trials for the national cholesterol education program adult treatment panel III guidelines. Circulation. 2004;110:227-239. doi:10.1161/01.CIR.0000133317.49796.0E

19. The National Institute for Health and Care Excellence (NICE). Hypertension: Clinical Management of Primary Hypertension in Adults. London: NICE; 2011.

20. Watanabe G, Hashimoto M, Matsuda M. Evaluation of new classification of Japanese gallbladder stones with chemical componential analysis. $J$ Stage. 2017;31(2):205-213.

21. Drossman DA. The functional gastrointestinal disorders and the Rome III process. Gastroenterology. 2006;130:1377-1390. doi:10.1053/j. gastro.2006.03.008

22. Chasalani N, Younossi Z, Lanvine JE, et al. The diagnostic and management of NAFLD: practice guidance from AASLD. Hepatology. 2018;67:328-357. doi:10.1002/hep.29367 
23. Shanahan F. The colonic microbiota in health and disease. Gastroenterology. 2013;29:49-54.

24. Brown R, Poxton IR, Wilkinson JF. Centrifuges, colorimeters and bacterial counts. In: Collee JG, Duguid JP, Fraser AG, Marmion BP, editors. Mackie and McCartney Practical Medical Microbiology. 14th ed. Edinburgh: Churchill Livingstone; 1999:200-247.

25. Sandrin TR, Goldstein JE, Shoemaker S. MALDI TOF MS profiling of bacteria at the strain level: a review. Mass Spectrom Rev. 2013;32(3):188. doi:10.1002/mas.21359

26. Ji B, Nielsen J. From next generation sequencing to systemic modeling of the gut microbiome. Front.Genet. 2015;6:219. doi:10.3389/ fgene.2015.00219

27. Strong WL. Biased richness and evenness relationships within Shannon-Wiener index values. Ecoll Ind. 2016;67:703-713. doi:10.1016/j. ecolind 2016.03 .043

28. Russello D, Di Stefano A, Scala R, et al. Does cholecystectomy always resolve biliary disease? Minerva Chir. 1997;52(12):1435-1439.

29. Radu D, Olariu S, Georgescu D, Teodorescu MS. Lost gallbladder stones in the peritoneal cavity after laparoscopic cholecystectomy. Surg Endosc. 2011;25(supp11):S54-148.

30. Gomes CA, Junior CS, Di Saverio S, et al. Acute calculous cholecystitis. Review of current best practice. WJG Surg. 2017;9(5):118-126. doi:10.4240/wjgs.v9.i5.118

31. Distrutti E, Monaldi L, Ricci P, Fiorucci S. Gut microbiota role in irritable bowel syndrome: new therapeutic strategies. WJG. 2016;22 (7):2219-2241. doi:10.3748/wjg.v22.i7.2219

32. Bhattarai Y, Muniz Pedrogo DA, Kashyap PC. Irritable bowel syndrome: a gut microbiota-related disorder? Am J Physiol Gastrointest Liver Physiol. 2017;312(1):G52-G62. doi:10.1152/ajpgi.00338.2016

33. Pittayanon R, Lau JT, Yuhong Y, et al. Gut microbiota in patients with irritable bowel syndrome-a systematic review. Gastroenterol. 2019;157:97-108. doi:10.1053/j.gastro.2019.03.049

34. Festi D, Schiumrini R, Eusebi LH, et al. Gut microbiota and metabolic syndrome. WJG. 2014;43(20):16079. doi:10.3748/wjg.v20.i43.16079

35. Belizario JE, Faintuch J, Garay-Malpartida M. Gut microbiome dysbiosis and immunometabolism: new frontiers for treatment of metabolic diseases. Med Infl. 2018;2018:2037838.

36. Jurca-Simina IE, Juganaru I, Iurciuc MS, et al. What if body fat percentage association with FINDRISC score leads to a better prediction of type 2 diabetes mellitus? Rom J Morphol Embryol. 2019;60(1):205-210.

37. Jin M, Qian Z, Yin J, Xu V, Zhou XJ. The role of intestinal microbiota in cardio-vascular disease. Cell Mol Med. 2019;4(23):2343. doi:10.1111/ jcmm. 14195

38. Zhuang X, Xiong L, Li L, Li M, Chen M. Alterations of gut microbiota in patients with irritable bowel syndrome: a systematic review and meta-analysis. J Gastroenterol Hepatol. 2017;32(1):28-38. doi:10.1111/jgh.13471

39. Georgescu D, Iurciuc MS, Petre I, et al. Chronic pelvic pain and irritable bowel syndrome: is subclinical inflammation bridging the gap? Revista de Chimie. 2019;70(10):3634-3637. doi:10.37358/RC.19.10.7611

40. Harris LA, Baffy N. Modulation of the gut microbiota: a focus on treatments for irritable bowel syndrome. Postgraduate Med. 2017;129 (8):872-888. doi:10.1080/00325481.2017.1383819

41. Li J, Zhao F, Wang Y, et al. Gut microbiota dysbiosis contributes to the development of hypertension. Microbiome. 2017;5:14. doi:10.1186/s40168016-0222-X

42. Kang Y, Cai Y. Gut microbiota and hypertension: from pathogenesis to new therapeutic strategies. Clin Res Hepatol Gastroenterol. 2018;42 (2):110-117. doi:10.1016/j.clinre.2017.09.006

43. Georgescu D, Iurciuc M, Ionita I, et al. Migraine without aura and subclinical atherosclerosis: is gut microbiota to blame? Med Lith. 2019;5 (12):786.

44. Junli M, Houkai L. The role of gut microbiota in atherosclerosis and hypertension. Front Pharmacol. 2018;9:1082. doi:10.3389/fphar.2018.01082

45. Georgescu D, Iurciuc M, Ionita I, et al. Portal vein thrombosis and gut microbiota: understanding the burdem. Revista de Chimie. 2019;70 (6):2181-2185. doi:10.37358/RC.19.6.7301

46. Wang Q, Jiao L, He C, et al. Alteration of gut microbiota in association with cholesterol gallstone formation in mice. BMC Gastroenterol. 2017;17:74. doi:10.1186/s12876-017-0629-2

47. Di Ciaula A, Wang DQ-H, Portincasa P. An update on the pathogenesis of cholesterol gallstone disease. Curr Opin Gastroenterol. 2018;34 (2):71-80. doi:10.1097/MOG.0000000000000423

48. Wang Q, Hao C, Yao W, et al. Intestinal flora imbalance affects bile acid metabolism and is associated with gallstone formation. BMC Gastroenterol. 2020;20:59. doi:10.1186/s12876-020-01195-1

49. De Filippo C, Cavalieri D, Di Paola M, et al. Impact of diet in shaping gut microbiota revealed by a comparative study in children from Europe and rural Africa. Proc Natl Acad Sci U S A. 2010;107(33):14691-14696. doi:10.1073/pnas.1005963107

50. Arumugam M, Raes J, Pelletier E, et al. Enterotypes of the human gut microbiome. Nature. 2011;473:174-180. doi:10.1038/nature09944

51. Robles-Alonso V, Guarner F. Progress in the knowledge of the intestinal human microbiota. Nutricion Hospitalaria. 2013;28(3):553-557. doi:10.3305/nh.2013.28.3.6601

52. Festi D, Schiumrini R, Eusebi LH, et al. Gut microbiota and metabolic syndrome. WJG. 2014;20(43):16079.

53. Zhang QQ, Lu LG. Nonalcoholic fatty liver disease: dyslipidemia, risk for cardio-vascular complications and treatment strategy. $J$ Clin Transl Hepatol. 2015;3(1):78.

54. Maqsood H, Patel K, Ferdosi H, et al. Age-related differences pre-, intra-, and postcholecystectomy: a retrospective cohort study of 6868 patients. Internat J Surg. 2017;39:119-126. doi:10.1016/j.ijsu.2017.01.046

55. Arora D, Kaushik R, Kaur R, Sachdev A. Post-cholecystectomy syndrome: a new look at an old problem. J Minim Access Surg. 2018;14 (3):202-207. doi:10.4103/jmas.JMAS_92_17

56. Forslund K, Hildebrand F, Nielsen T, et al. Disentangling type 2 diabetes and metformin treatment signatures in the human gut microbiota. Nature. 2015;28(7581):262. doi:10.1038/nature15766

57. Saltzman ET, Palacios T, Thomsen M, Vitetta L. Intestinal microbiome shifts, dysbiosis, inflammation and nonalcoholic fatty liver disease. Front Microbiol. 2018;9:61. doi:10.3389/fmicb.2018.00061

58. Wang W, Wang J, Li J, et al. Cholecystectomy damages aging-associated intestinal microbiota construction. Front Microbiol. 2018;9:1402. doi:10.3389/fmicb.2018.01402 
59. Ren X, Xu J, Zhang Y, et al. Bacterial alterations in post-cholecystectomy patients are associated with colorectal cancer. Front Oncol. 2020;10:1418. doi:10.3389/fonc.2020.01418

60. Zhang Y, Yu X, Yu E, et al. Changes in gut microbiota and plasma inflammatory factors across the stages of colorectal tumorigenesis: a case-control study. BMC Microbiol. 2018;18:92. doi:10.1186/s12866-018-1232-6

61. Vacante M, Ciuni R, Basile F, Biondi A. Gut microbiota and colorectal cancer development: a closer look to the adenoma-carcinoma sequence. Biomed. 2020;8:489.

62. Jaime R, Guarner F, Bustos FL, Maruy A, Sdepanian VL, Cohen H. Antibiotics as major disruptors of gut microbiota. Front Cell Infect Microbiol. 2020;10:731.

63. Strati F, Pujolassos M, Burrello C, et al. Antibiotic-associated dysbiosis affects the ability of the gut microbiota to control intestinal inflammation upon fecal microbiota transplantation in experimental colitis models. Microbiome. 2021;9:39. doi:10.1186/s40168-020-00991

64. Radu D, Georgescu D, Teodorescu M. Diet and postcholecystectomy syndrome (PCS). J Agroaliment Process Technol. $2012 ; 18: 219-222$.

65. Shirah BH, Shirah HA, Zafar SH, Albeladi KB. Clinical patterns of postcholecystectomy syndrome. Ann Hepatobiliary Pancreat Surg. 2018;22:52-57. doi:10.14701/ahbps.2018.22.1.52

66. Isherwood J, Oakland K, Khanna A. A systematic review of the aetiology and management of post cholecystectomy syndrome. Surgeon. 2019;17 (1):33-42. doi:10.1016/j.surge.2018.04.001

67. Yoon WJ, Kim HN, Park E, et al. The impact of cholecystectomy on the gut microbiota: a case-control study. J Clin Med. 2019;8(1):79. doi: $10.3390 / \mathrm{jcm} 8010079$

\section{Publish your work in this journal}

The International Journal of Women's Health is an international, peer-reviewed open-access journal publishing original research, reports, editorials, reviews and commentaries on all aspects of women's healthcare including gynecology, obstetrics, and breast cancer. The manuscript management system is completely online and includes a very quick and fair peer-review system, which is all easy to use. Visit http://www. dovepress.com/testimonials.php to read real quotes from published authors.

Submit your manuscript here: https://www.dovepress.com/international-journal-of-womens-health-journal 